\title{
BICOVARIANT DIFFERENTIAL CALCULI AND CROSS PRODUCTS ON BRAIDED HOPF ALGEBRAS
}

\author{
YURI BESPALOV \\ National Academy of Sciences \\ Bogolyubov Institute for Theoretical Physics \\ 252 143, Kiev-143, Ukraine \\ E-mail: mmtpitp@gluk.apc.org \\ BERNHARD DRABANT* \\ Department of Mathematics, Katholieke Universiteit Leuven \\ Celestijnenlaan 200B, 3001 Leuven-Heverlee, Belgium \\ E-mail:drabant@davinci.wis.kuleuven.ac.be
}

\begin{abstract}
In a braided monoidal category $\mathcal{C}$ we consider Hopf bimodules and crossed modules over a braided Hopf algebra $H$. We show that both categories are equivalent. It is discussed that the category of Hopf bimodule bialgebras coincides up to isomorphism with the category of bialgebra projections over $H$. Using these results we generalize the Radford-Majid criterion and show that bialgebra cross products over the Hopf algebra $H$ are precisely described by $H$-crossed module bialgebras. In specific braided monoidal abelian categories we define (bicovariant) braided differential calculi over $H$ and apply the results on Hopf bimodules to construct a higher order bicovariant differential calculus over $H$ out of any first order bicovariant differential calculus over $H$. This object is shown to be a bialgebra with universal properties.

1. Introduction. Cross products and cross coproducts of $k$-bialgabras over a field $k$ have been studied in [17]. Let $H$ be a Hopf algebra and $X$ be an $H$-right module algebra and an $H$-right comodule coalgebra. Then the conditions are derived for $H \otimes X$ to be a bialgebra such that the algebra structure is given by the cross product and the coalgebra structure is given by the cross coproduct. In this case Radford calls $(H, X)$ an admissible pair or simply bialgebra cross product over $H$ [17]. The possibility of forming bialgebra cross products over a quasitriangular Hopf algebra with any of its modules

1991 Mathematics Subject Classification: Primary 16W30, 17B37; Secondary 18D10, 81R50.

*Address after Jaunary 1997: Department of Theoretical Physics, University of Valencia, E-46100 Burjassot (Valencia), Spain, e-mail: drabant@lie.uv.es.

The paper is in final form and no version of it will be published elsewhere.
\end{abstract}


has been recovered in [11]. In Radford's definition of an admissible pair crossed modules implicitely appear. Explicitely crossed modules are defined in [21, 18]. Majid proved that the category of crossed modules is braided monoidal [10]. He recovered that the (co-) modules to be candidates for a bialgebra cross product are crossed module bialgebras and hence he formulated the Radford criterion on bialgebra cross products in terms of crossed module bialgebras.

Bicovariant differential calculi over $k$-Hopf algebras have been investigated in [20]. The so-called bicovariant bimodules [20] or Hopf bimodules are of special importance for the construction of bicovariant differential calculi. The equivalent description of Hopf bimodules through crossed modules has been found in [20].

In $[1,2]$ crossed modules and Hopf (bi-)modules in braided monoidal categories are constructed. The equivalence of braided crossed modules and braided Hopf bimodules has been found in [2] as well as the isomorphy of the braided Hopf bimodule bialgebras and the braided bialgebra projections over a Hopf algebra $H$ with invertible antipode. Both theorems can be applied to generalize the Radford-Majid criterion to braided categories. In the special case of braided quasitriangular Hopf algebras $\mathcal{H}[11]$ this construction works for any $\mathcal{H}$-module Hopf algebra in a specific admissible category of $\mathcal{H}$-modules $[1,5]$. As a result inhomogeneous quantum groups without additional dilaton generator have been constructed [5].

Another application of the central theorems of [2] in certain braided abelian categories is the construction of a graded Hopf algebra differential calculus over $H$ which is in some sense uniquely derived from a given braided bicovariant first order differential calculus. This result is discussed in [3] and is an extension of the results of [20] to braided categories.

The paper in hand is concerned with these subjects. It is mainly based on $[2,3]$. In Section 2 we fix our conventions and give the necessary definitions needed in the following. We state the central results of [2]. Section 3 is dedicated to cross product constructions. We formulate the generalized braided Radford-Majid criterion and consider cross products over braided quasitriangular Hopf algebras or braided quantum groups. In Section 4 we restrict to certain braided abelian monoidal categories which we call $* *-$ abelian, and define (bicovariant) differential calculi in a generalized form. Staring from a bicovariant first order differential calculus over a braided Hopf algebra $H$ with invertible antipode we deduce a graded Hopf algebra differential calculus over $H$. We outline the universality of this graded bialgebra. For the derivation of these results extensive use of the results of Section 2 has to be made.

2. Braided crossed modules and Hopf bimodules. For the definition of a monoidal category we refer to [14]. From Mac Lane's coherence theorem [15] it is known that every monoidal category is equivalent to a strict one. Hence we restrict most of the considerations to strict monoidal categories and denote by $\mathcal{C}:=(\mathcal{C}, \otimes, \mathbf{1}, \Psi)$ a braided monoidal category $[6,9]$ where $\otimes$ is the tensor product (bifunctor), $\mathbf{1}$ is the unit object and $\Psi$ is the braiding. We suppose that the reader is familiar with the notion of (co-)associative (co-)unital (co-)algebras, (co-)modules and bi-(co-)modules in monoidal categories [19, $11,10]$. In a braided monoidal category the tensor product of two (co-)algebras is again a (co-)algebra; the multiplication $\mathrm{m}_{U \otimes V}$ and the unit $\eta_{U \otimes V}$ of two algebras $\left(U, \mathrm{~m}_{U}, \eta_{U}\right)$ 
and $\left(V, \mathrm{~m}_{V}, \eta_{V}\right)$ are given through $\mathrm{m}_{U \otimes V}=\left(\mathrm{m}_{U} \otimes \mathrm{m}_{V}\right) \circ\left(\mathrm{id}_{U} \otimes \Psi_{U, V} \otimes \mathrm{id}_{V}\right)$ and $\eta_{U \otimes V}=\eta_{U} \otimes \eta_{V}$ respectively. The coalgebra structure of the tensor product of two coalgebras is obtained in the dual symmetric manner which means that the order of the composition of morphisms will be reversed, and the multiplication $\mathrm{m}$ will be replaced by the comultiplication $\Delta$ and the unit $\eta$ by the counit $\varepsilon$, and vice versa. A bialgebra $(B, \mathrm{~m}, \eta, \Delta, \varepsilon)$ in a braided monoidal category $\mathcal{C}$ is an algebra $(B, \mathrm{~m}, \eta)$ and a coalgebra $(B, \Delta, \varepsilon)$ where $\Delta$ and $\varepsilon$ are algebra morphisms [10]. A Hopf algebra $(H, \mathrm{~m}, \eta, \Delta, \varepsilon, S)$ in $\mathcal{C}$ is a bialgebra together with the antipode $S: H \rightarrow H$ such that $\operatorname{mo}\left(\operatorname{id}_{H} \otimes S\right) \circ \Delta=\operatorname{m\circ }\left(S \otimes \operatorname{id}_{H}\right) \circ \Delta=\eta \circ \varepsilon$. Every bialgebra $(B, \mathrm{~m}, \eta, \Delta, \varepsilon)$ in $\mathcal{C}$ is a bi-(co-)module through the regular action $\mathrm{m}$ and the regular coaction $\Delta$. The diagonal action of the tensor product of two right modules $\left(X, \mu_{r}^{X}\right)$ and $\left(Y, \mu_{r}^{Y}\right)$ is given by $\mu_{d, r}^{X \otimes Y}=\left(\mu_{r}^{X} \otimes \mu_{r}^{Y}\right) \circ\left(\operatorname{id}_{X} \otimes \Psi_{Y B} \otimes \operatorname{id}_{B}\right) \circ\left(\operatorname{id}_{X} \otimes \operatorname{id}_{Y} \otimes \Delta\right)$. The right action $\mu_{i, r}$ on $X \otimes Y$ induced by $Y$ is given through $\mu_{i, r}^{X \otimes Y}=\mathrm{id}_{X} \otimes \mu_{r}^{Y}$. (Dually) analogue all other types of diagonal (co-)actions and induced (co-)actions are defined. We will also use the notion of (co-)adjoint (co-)action. Let $H$ be a Hopf algebra in $\mathcal{C}$ and let $\left(X, \mu_{r}, \mu_{l}\right)$ be an $H$-bimodule then $X$ becomes a right $H$-module through the right adjoint action ad $\triangleleft:=\mu_{l} \circ\left(\operatorname{id}_{H} \otimes \mu_{r}\right) \circ\left(\Psi_{X H} \otimes \operatorname{id}_{H}\right) \circ\left(\operatorname{id}_{X} \otimes\left(S \otimes \operatorname{id}_{H}\right) \circ \Delta\right)$. Similarly the left adjoint action is defined. The coadjoint coactions are obtained in the dual symmetric manner. If $A$ is an algebra and $f: H \rightarrow A$ is an algebra morphism then the algebra $A$ becomes an $H$-bimodule $\left(A, \mu_{r}^{f}, \mu_{l}^{f}\right)$ via pullback along $f, \mu_{l}^{f}=\mathrm{m}_{A} \circ\left(f \otimes \mathrm{id}_{A}\right)$ and $\mu_{r}^{f}=\mathrm{m}_{A} \circ\left(\operatorname{id}_{A} \otimes f\right)$. The corresponding right adjoint action (induced by $f$ ) will be denoted by $\operatorname{ad}_{f} \triangleleft$ and the resulting right $H$-module algebra by $A_{f}:=\left(A, \mathrm{~m}, \eta, \mathrm{ad}_{f} \triangleleft\right)$.

We suppose that the categories $\mathcal{C}$ admit split idempotents, i.e. every idempotent in the category $\mathcal{C}, \mathrm{e}=\mathrm{e}^{2}: X \rightarrow X$ splits in $\mathcal{C}$ in the sense that there exists an object $X_{\mathrm{e}}$ and morphisms $\mathrm{i}_{\mathrm{e}}: X_{\mathrm{e}} \rightarrow X$ and $\mathrm{p}_{\mathrm{e}}: X \rightarrow X_{\mathrm{e}}$ such that $\mathrm{e}=\mathrm{i}_{\mathrm{e}} \circ \mathrm{p}_{\mathrm{e}}$ and $\mathrm{id}_{\mathrm{e}}=\mathrm{p}_{\mathrm{e}} \circ \mathrm{i}_{\mathrm{e}}$. For example this holds in every abelian category. In what follows $B$ and $H$ denote bialgebras and Hopf algebras in the braided category $\mathcal{C}$ respectively. We assume that the antipode is invertible. The remainder of this section is mostly based on the results of $[1,2]$.

We start with the definition of braided crossed modules. They are (braided) analogues of the $k$-vector space of invariant one-forms of a bicovariant bimodule (Hopf bimodule) [20] and have a close connection to smash product constructions (see [17] in the case of bialgebras over the field $k$ and [1] for the braided case).

Definition 1. A right crossed module $\left(X, \mu_{r}, \nu_{r}\right)$ over the bialgebra $B$ is a right $B$ module and a right $B$-comodule obeying the compatibility relations

$$
\begin{aligned}
& \left(\operatorname{id}_{X} \otimes m\right) \circ\left(\Psi_{B X} \otimes \operatorname{id}_{B}\right) \circ\left(\operatorname{id}_{B} \otimes \nu_{r} \circ \mu_{r}\right) \circ\left(\Psi_{X B} \otimes B\right) \circ\left(\operatorname{id}_{X} \otimes \Delta\right) \\
& =\left(\mu_{r} \otimes m\right) \circ\left(\operatorname{id}_{X} \otimes \Psi_{B B} \otimes \operatorname{id}_{B}\right) \circ\left(\nu_{r} \otimes \Delta\right) .
\end{aligned}
$$

The category $\mathcal{D} \mathcal{Y}(\mathcal{C})_{B}^{B}$ is the category of crossed modules where the morphisms are both right module and right comodule morphisms over $B$. In a similar way all other combinations of crossed modules will be defined.

EXAMPLE 1. A Hopf algebra $H$ is a crossed module over itself through the adjoint action and the regular coaction. Dually analogue $H$ is a crossed module through the regular action and the coadjoint coaction. 
The (pre-)braided monoidal structure of $\mathcal{D} \mathcal{Y}(\mathcal{C})_{B}^{B}$ is described in the next theorem.

THEOREM 1. For the bialgebra $B$ in $\mathcal{C}$ the category $\left(\mathcal{D Y}(\mathcal{C})_{B}^{B}, \otimes, \mathbf{1}\right)$ is monoidal with unit object $\left(\mathbf{1}, \varepsilon_{B}, \eta_{B}\right)$. It is pre-braided through

$$
{ }^{\mathcal{D Y}(\mathcal{C})}{ }_{B}^{B} \Psi_{X Y}:=\left(\operatorname{id}_{Y} \otimes \mu_{r}^{X}\right) \circ\left(\Psi_{X Y} \otimes \operatorname{id}_{B}\right) \circ\left(\operatorname{id}_{X} \otimes \nu_{r}^{Y}\right)
$$

where $X, Y \in \operatorname{Ob}\left(\mathcal{D Y}(\mathcal{C})_{B}^{B}\right)$. If $H$ is a Hopf algebra (with isomorphic antipode) in $\mathcal{C}$ then $\mathcal{D Y}(\mathcal{C})_{H}^{H}$ is braided, i.e. the inverse of (2) exists and equals

$$
\begin{aligned}
\left({ }^{\mathcal{D} \mathcal{Y}(\mathcal{C})}{ }_{B}^{B} \Psi_{X Y}\right)^{-1}= & \left(\mu_{r}^{X} \otimes \operatorname{id}_{Y}\right) \circ\left(\operatorname{id}_{Y} \otimes\left(\Psi_{H Y}\right)^{-1}\right) \circ \\
& \left(\left(\Psi_{X Y}\right)^{-1} \otimes S^{-1}\right) \circ\left(\operatorname{id}_{Y} \otimes\left(\Psi_{X H}\right)^{-1}\right) \circ\left(\nu_{r}^{Y} \otimes \operatorname{id}_{X}\right)
\end{aligned}
$$

Hopf bimodules in the braided category $\mathcal{C}$ are defined as follows.

Definition 2. An object $\left(X, \mu_{r}, \mu_{l}, \nu_{r}, \nu_{l}\right)$ is called a $B$-Hopf bimodule in $\mathcal{C}$ if $\left(X, \mu_{r}, \mu_{l}\right)$ is a $B$-bimodule, and $\left(X, \nu_{r}, \nu_{l}\right)$ is a $B$-bicomodule in the category of $B$ bimodules, where the regular (co-)action on $B$ and the diagonal (co-)action on tensor products of modules are used. Hopf bimodules together with the $B$-bimodule-bicomodule morphisms form the category of Hopf bimodules which will be denoted by ${ }_{B}^{B} \mathcal{C}_{B}^{B}$.

A consequence of the bimodule property is given by the following lemma.

Lemma 2. Let $\left(X, \mu_{r}, \mu_{l}, \nu_{r}, \nu_{l}\right)$ be an H-Hopf bimodule. Then the morphism ${ }_{X} \Pi$ : $X \rightarrow X$ defined through ${ }_{X} \Pi:=\mu_{l} \circ\left(S \otimes \mathrm{id}_{X}\right) \circ \nu_{l}$ is an idempotent.

We denote by ${ }_{X} \mathrm{i}:{ }_{H} X \rightarrow X,{ }_{X} \mathrm{p}: X \rightarrow{ }_{H} X$ the morphisms which split the idempotent ${ }_{X} \Pi$, i.e. ${ }_{X}{ }^{\mathrm{i} \circ}{ }_{X} \mathrm{p}={ }_{X} \Pi$ and ${ }_{X} \mathrm{p} \circ_{X} \mathrm{i}=\mathrm{id}_{H}$. Then the assignment ${ }_{H}(-):{ }_{H}^{H} \mathcal{C}_{H}^{H} \rightarrow \mathcal{D} \mathcal{Y}(\mathcal{C})_{H}^{H}$ which is given through ${ }_{H}(X):={ }_{H} X$ for an $H$-Hopf bimodule $X$, and through ${ }_{H}(f)=$ $Y$ p $\circ \circ_{X}$ i for a Hopf bimodule morphism $f: X \rightarrow Y$, defines a functor. The right crossed module structure on ${ }_{H} X$ is given by $\mu_{r}^{H} X={ }_{X} \mathrm{p} \circ \mu_{r}^{X} \circ\left({ }_{X} \mathrm{i} \otimes \mathrm{id}_{H}\right)$ and $\nu_{r}^{H}=\left({ }_{X} \mathrm{p} \otimes \mathrm{id}_{H}\right) \circ$ $\nu_{r}^{X} \circ{ }_{H}$ i. Conversely if $Y$ is a right crossed module over $H$ then a full inclusion functor $H \ltimes(-): \mathcal{D} \mathcal{Y}(\mathcal{C})_{H}^{H} \rightarrow{ }_{H}^{H} \mathcal{C}_{H}^{H}$ is defined by $H \ltimes(Y)=\left(H \otimes Y, \mu_{i, l}^{H \otimes Y}, \nu_{i, l}^{H \otimes Y}, \mu_{d, r}^{H \otimes Y}, \nu_{d, r}^{H \otimes Y}\right)$ and $H \ltimes(f)=\operatorname{id}_{H} \otimes f$ for any crossed module morphism $f$. These facts will be used to formulate the following important theorems.

THEOREM 3. The category of Hopf bimodules over $H$ is monoidal with the tensor product given by

$$
\otimes_{H}:=\otimes \circ\left(\mathrm{id} \times{ }_{H}(-)\right):{ }_{H}^{H} \mathcal{C}_{H}^{H} \times{ }_{H}^{H} \mathcal{C}_{H}^{H} \longrightarrow{ }_{H}^{H} \mathcal{C}_{H}^{H} .
$$

$H$ is the unit object. It is equipped with the regular $H$-Hopf bimodule structure. ${ }_{H}^{H} \mathcal{C}_{H}^{H}$ is braided with the braiding given on the objects $X, Y \in \mathrm{Ob}\left({ }_{H}^{H} \mathcal{C}_{H}^{H}\right)$ through

$$
{ }_{H}^{H}{ }^{\mathcal{C}_{H}^{H}} \Psi_{X Y}=\left(\mu_{l}^{Y} \otimes{ }_{X} \mathrm{p} \circ \mu_{r}^{X}\right) \circ\left(\operatorname{id}_{H} \otimes \Psi_{X Y} \otimes \operatorname{id}_{H}\right) \circ\left(\nu_{l}^{X} \otimes \nu_{r}^{Y} \circ{ }_{Y} \mathrm{i}\right) .
$$

THEOREM 4. Let $H$ be a Hopf algebra in $\mathcal{C}$ with isomorphic antipode. Then the categories $\mathcal{D} \mathcal{Y}(\mathcal{C})_{H}^{H}$ and ${ }_{H}^{H} \mathcal{C}_{H}^{H}$ are braided monoidal equivalent through the functors $\mathcal{D Y}(\mathcal{C})_{H}^{H} \underset{H(-)}{\stackrel{H \ltimes(-)}{\longrightarrow}}{ }_{H} \mathcal{C}_{H}^{H}$. 
3. Braided cross products. In this section we define braided cross products and generalize the Radford-Majid criterion to braided categories. We review braided quasitriangular Hopf algebras and study braided quasitriangular bialgebra cross products. (See $[1,2,5])$

For the investigation of cross product Hopf algebras, Hopf algebra projections and Hopf bimodule Hopf algebras we introduce the relative antipode of a Hopf bimodule.

Definition 3. Let $\left(X, \mu_{r}, \mu_{l}, \nu_{r}, \nu_{l}\right)$ be an $H$-Hopf bimodule. Then the relative antipode $S_{X / H}$ of $X$ w.r.t. $H$ is defined by

$$
S_{X / H}:=M_{X} \circ\left(S \otimes \operatorname{id}_{X} \otimes S\right) \circ N_{X}
$$

where $M_{X}:=\mu_{l} \circ\left(\operatorname{id}_{H} \otimes \mu_{r}\right)$ and $N_{X}:=\left(\operatorname{id}_{H} \otimes \nu_{r}\right) \circ \nu_{l}$.

A "polarized" form of the anti-(co-)multiplicity of the antipode holds, $S_{X / H} \circ \mu_{r}=$ $\mu_{l} \circ \Psi_{X H} \circ\left(S_{X / H} \otimes S\right)$ and $S_{X / H} \circ \mu_{l}=\mu_{r} \circ \Psi_{H X} \circ\left(S \otimes S_{X / H}\right)$, and dually analogue for the coactions. The relative antipode $S_{H / H}$ coincides with the antipode $S$ of $H$. In the next definition we define braided bialgebra projections in the sense of [17].

Definition 4 . Let $B_{1}$ and $B_{2}$ be bialgebras in $\mathcal{C}$ and $B_{1} \stackrel{\eta}{\rightarrow} B_{2} \stackrel{\varepsilon}{\rightarrow} B_{1}$ be a pair of bialgebra morphisms such that $\underline{\varepsilon} \circ \underline{\eta}=\mathrm{id}_{B_{1}}$. Then $\left(B_{1}, B_{2}, \underline{\eta}, \underline{\varepsilon}\right)$ is called a bialgebra projection on $B_{1}$. The bialgebra projections on $B_{1}$ constitute the category $B_{1}$-Bialg-C. The morphisms obey the relations $f \circ \underline{\eta}=\underline{\eta}$ and $\underline{\varepsilon} \circ f=\underline{\varepsilon}$.

One easily verifies that for a bialgebra projection $\left(B_{1}, B_{2}, \underline{\eta}, \underline{\varepsilon}\right)$ on $B_{1}$ the object $\underline{B}_{2}=\left(B_{2}, \mu_{r}^{B_{2}}, \mu_{l}^{B_{2}}, \nu_{r}^{B_{2}}, \nu_{l}^{B_{2}}\right)$ is a $B_{1}$-Hopf bimodule through the (co-)actions

$$
\begin{array}{ll}
\mu_{l}^{B_{2}}=\mathrm{m}_{B_{2}} \circ\left(\underline{\eta} \otimes \mathrm{id}_{B_{2}}\right), & \mu_{r}^{B_{2}}=\mathrm{m}_{B_{2}} \circ\left(\operatorname{id}_{B_{2}} \otimes \underline{\eta}\right), \\
\nu_{l}^{B_{2}}=\left(\underline{\varepsilon} \otimes \mathrm{id}_{B_{2}}\right) \circ \Delta_{B_{2}}, & \nu_{r}^{B_{2}}=\left(\operatorname{id}_{B_{2}} \otimes \underline{\varepsilon}\right) \circ \Delta_{B_{2}} .
\end{array}
$$

For the formulation of the next theorem observe that in the category ${ }_{H}^{H} \mathcal{C}_{H}^{H}$ the tensor product $\underset{H}{\otimes}$ and the cotensor product $\square_{H}$ exist and coincide up to isomorphism with $\otimes_{H}$. The corresponding coequalizer $\lambda$ and equalizer $\rho$ for two $H$-Hopf bimodules $N$ and $M$ are given respectively by

$$
\begin{aligned}
& N \otimes M \stackrel{\lambda_{N, M}^{H}}{\longrightarrow} N \underset{H}{\otimes} M \cong N \otimes{ }_{H} M \\
& \lambda_{N, M}^{H}=\left(\mu_{r}^{N} \otimes \operatorname{id}_{H} M\right) \circ\left(\operatorname{id}_{N} \otimes\left(\operatorname{id}_{H} \otimes_{M} \mathrm{p}\right) \circ \nu_{l}^{M}\right)
\end{aligned}
$$

and

$$
\begin{aligned}
& N \otimes_{H} M \cong N \underset{H}{\square} M \stackrel{\rho_{N, M}^{H}}{\longrightarrow} N \otimes M \\
& \rho_{N, M}^{H}=\left(\operatorname{id}_{N} \otimes \mu_{l}^{M} \circ\left(\operatorname{id}_{H} \otimes_{M} \mathrm{i}\right) \circ\left(\nu_{r}^{N} \otimes \mathrm{id}_{H} M\right) .\right.
\end{aligned}
$$

The following theorem states that the categories of Hopf bimodule bialgebras and bialgebra projections are equal up to isomorphism.

TheOrem 5. For any bialgebra projection $\left(H, B, \underline{\eta}_{B}, \underline{\varepsilon}_{B}\right)$ the object $\underline{B}$ is a bialgebra $\left(\underline{B}, \underline{\mathrm{m}}_{B}, \underline{\eta}_{B}, \underline{\Delta}_{B}, \underline{\varepsilon}_{B}\right)$ in ${ }_{H}^{H} \mathcal{C}_{H}^{H}$ where the projection morphisms $\underline{\eta}_{B}$ and $\underline{\varepsilon}_{B}$ are the unit and 
counit respectively. Multiplication $\underline{\mathrm{m}}_{B}$ and comultiplication $\underline{\Delta}_{B}$ are defined through

$$
\underline{\mathrm{m}}_{B}=\mathrm{m}_{B} \circ\left(\mathrm{id}_{B} \otimes_{H} \mathrm{i}\right) \text { and } \underline{\Delta}_{B}=\left(\mathrm{id}_{B} \otimes_{H} \mathrm{p}\right) \circ \Delta \text {. }
$$

Conversely every bialgebra $\underline{B}=\left(B, \underline{\mathrm{m}}_{B}, \underline{\eta}_{B}, \underline{\Delta}_{B}, \underline{\varepsilon}_{B}\right)$ in ${ }_{H}^{H} \mathcal{C}_{H}^{H}$ can be turned into a bialgebra $B=\left(B, \mathrm{~m}_{B}, \eta_{B}, \Delta_{B}, \varepsilon_{B}\right)$ in $\mathcal{C}$ where the structure morphisms are given by

$$
\mathrm{m}_{B}=\underline{\mathrm{m}}_{B} \circ \lambda_{B, B}^{H}, \quad \eta_{B}=\underline{\eta}_{B} \circ \eta_{H}, \quad \Delta_{B}=\rho_{B, B}^{H} \circ \underline{\Delta}_{B}, \quad \varepsilon_{B}=\varepsilon_{H} \circ \underline{\varepsilon}_{B} .
$$

The pair $(H, B)$ is a bialgebra projection on $H$ via the morphisms $\left(\eta_{B}, \underline{\varepsilon}_{B}\right)$. There also exists a correspondence of Hopf algebra structures. For a Hopf algebra projection $\left(H_{1}, H_{2}, \underline{\eta}_{H_{2}}, \underline{\varepsilon}_{H_{2}}\right)$ on $H_{1}$ the antipode of $\underline{H}_{2}$ is given by

$$
\underline{S}_{H_{2}}=M_{H_{2}} \circ\left(\operatorname{id}_{H_{1}} \otimes S_{H_{2}} \otimes \operatorname{id}_{H_{1}}\right) \circ N_{H_{2}}
$$

and for any Hopf algebra $\underline{H_{2}}$ in ${ }_{H_{1}}^{H_{1}} \mathcal{C}_{H_{1}}^{H_{1}}$ the antipode of $H_{2}$ is given by

$$
S_{H_{2}}=\underline{S}_{H_{2}} \circ S_{H_{2} / H_{1}}=S_{H_{2} / H_{1}} \circ \underline{S}_{H_{2}} .
$$

In other words the categories $H$-Bialg- $\mathcal{C}$ and Bialg- ${ }_{H}^{H} \mathcal{C}_{H}^{H}$ are isomorphic through the functorial assignment on the objects described in equations (9) - (12) and through the identity on the morphisms.

The equivalence of the braided categories ${ }_{H}^{H} \mathcal{C}_{H}^{H}$ and $\mathcal{D} \mathcal{Y}(\mathcal{C})_{H}^{H}$ allows us to modify the previous theorem in terms of the category $\mathcal{D} \mathcal{Y}(\mathcal{C})_{H}^{H}$. Then we obtain a formulation of bialgebra cross products in terms of braided crossed module bialgebras and a braided generalization of the Radford-Majid criterion (see $[17,10]$ and $[1,2]$ in the braided case). This will be outlined in more detail in the following.

The braided cross product and the braided cross coproduct in the category $\mathcal{C}$ can be defined in a formal manner similar to the symmetric case. For a Hopf algebra $H$ in $\mathcal{C}$ and an algebra $\left(A, \mu_{A}\right)$ in $\mathcal{C}_{H}$ the cross product $H \ltimes_{\mu_{A}} A$ is defined as the universal algebra in $\mathcal{C}$ such that:

1. There are algebra morphisms j : $H \rightarrow H \ltimes_{\mu_{A}} A$ and i : $A \rightarrow H \ltimes_{\mu_{A}} A$. In addition $\mathrm{i}$ is algebra morphism in $\mathcal{C}_{H}$ where the module structure on $H \ltimes_{\mu_{A}} A$ is the right adjoint action induced by the morphism j, i.e. $\mathrm{i} \in \operatorname{Alg}_{H}\left(A,\left(H \ltimes_{\mu_{A}} A\right)_{\mathrm{j}}\right)$

2. If $U$ is any algebra in $\mathcal{C}$ and $g \in \operatorname{Alg}(H, U), f \in \operatorname{Alg}_{H}\left(A, U_{g}\right)$, then there exists a unique algebra morphism $g \ltimes_{\mu_{A}} f: H \ltimes_{\mu_{A}} A \rightarrow U$ such that $f=\left(g \ltimes_{\mu_{A}} f\right) \circ \mathrm{i}$ and $g=\left(g \ltimes_{\mu_{A}} f\right) \circ \mathrm{j}$.

The cross coproduct $H \ltimes^{\nu_{C}} C$ is defined in the dual symmetric manner. Both products are unique up to (co-)algebra isomorphism. The cross product can be realized on the tensor product $H \otimes A$ through $[11,5,2]$

$$
\begin{gathered}
\mathrm{m}_{\ltimes}=\left(\mathrm{m}_{H} \otimes \mathrm{m}_{A}\right) \circ\left(\mathrm{id}_{H} \otimes\left(\mathrm{id}_{H} \otimes \mu_{r}^{A}\right) \circ\left(\Psi_{A H} \otimes \mathrm{id}_{H}\right) \circ\left(\mathrm{id}_{A} \otimes \Delta_{H}\right) \otimes \mathrm{id}_{A}\right), \\
\eta_{\ltimes}=\eta_{H} \otimes \eta_{A}, \quad \mathrm{i}=\eta_{H} \otimes \mathrm{id}_{A}, \quad \mathrm{j}=\mathrm{id}_{H} \otimes \eta_{A} .
\end{gathered}
$$

Then $g \ltimes_{\mu_{A}} f=\mathrm{m}_{U} \circ(g \otimes f)$ for the corresponding unique morphism which is induced by $g$ and $f$.

If $X$ is at the same time a right $H$-module algebra and a right $H$-comodule coalgebra such that the smash product and the smash coproduct (realized on $H \otimes X$ ) are compatible in such a way that $H \ltimes X:=\left(H \otimes X, \mathrm{~m}_{\ltimes}, \eta_{\ltimes}, \Delta_{\ltimes}, \varepsilon_{\ltimes}\right)$ is a bialgebra in $\mathcal{C}$ then we say, in 
the sense of [17], that the pair $(H, X)$ is admissible; $X$ is called $H$-admissible object in $\mathcal{C}$. The category $H$-cp-C is the category of admissible pairs $(H, X)$ with bialgebra morphisms $h: H \ltimes X \rightarrow H \ltimes Y$ such that $h \circ \mathrm{j}_{X}=\mathrm{j}_{Y}$ and $\mathrm{k}_{Y} \circ h=\mathrm{k}_{X}$, where $\mathrm{k}=\varepsilon \otimes \mathrm{id}_{H}$.

Without difficulties one proofs that the following relations hold for an admissible pair $(H, X)$ in $\mathcal{C}$.

$$
\begin{array}{ll}
\varepsilon_{X} \circ \mathrm{m}_{X}=\varepsilon_{X} \otimes \varepsilon_{X}, & \varepsilon_{X} \circ \mu_{r}^{X}=\varepsilon_{X} \otimes \varepsilon_{H}, \\
\Delta_{X} \circ \eta_{X}=\eta_{X} \otimes \eta_{X}, & \nu_{r}^{X} \circ \eta_{X}=\eta_{X} \otimes \eta_{H}, \\
\varepsilon_{X} \circ \eta_{X}=\mathrm{id}_{\mathbf{1}} &
\end{array}
$$

From (13) it follows that $\left(H, H \ltimes X, \mathrm{id}_{H} \otimes \eta_{X}, \mathrm{id}_{H} \otimes \varepsilon_{X}\right)$ is a bialgebra projection on $H$. Using the results of [1] and Theorems 4 and 5 this yields a description of $H$-admissible objects in the category $\mathcal{C}$ in terms of crossed module bialgebras.

TheOREm 6. Let $H$ be a Hopf algebra in $\mathcal{C}$ with isomorphic antipode. Then the category of admissible pairs $H$-cp- $\mathcal{C}$ and the category of $H$-crossed module bialgebras

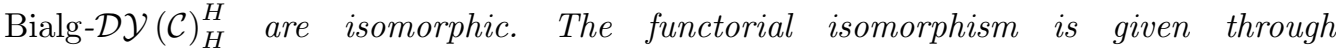

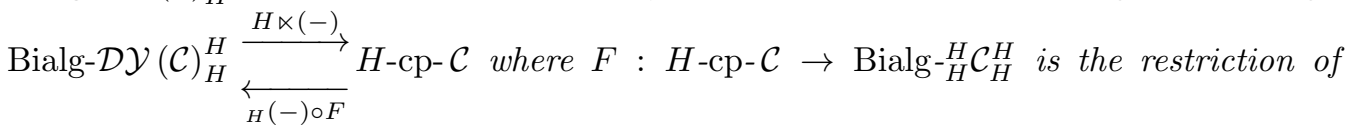
the corresponding isomorphism of Theorem 5.

Braided quantum groups (quasitrangular Hopf algebras) were introduced in [12] and the basic theory was developed there. We use slightly modified definitions [1] which reflect the symmetry between the two coalgebra structures under consideration. In what follows we set $\overline{\mathcal{C}}:=\left(\mathcal{C}, \otimes, \mathbf{1}, \Psi^{-1}\right)$, which is the same monoidal category as $\mathcal{C}$ but with inverse braiding. Then we say that a pair of bialgebras (Hopf algebras) $(A, \bar{A})$ in $\mathcal{C} \times \overline{\mathcal{C}}$ and a convolution invertible bialgebra pairing $\mathcal{R}: \mathbf{1} \rightarrow \bar{A}_{\mathrm{op}} \otimes A$ in $\mathcal{C}$ define a braided quasitriangular bialgebra (Hopf algebra) $(A, \bar{A}, \mathcal{R})$, if $A$ and $\bar{A}$ only differ in their comultiplication (and antipode), i.e. $A=(A, \mathrm{~m}, \eta, \Delta, \varepsilon(, S))$ and $\bar{A}=(A, \mathrm{~m}, \eta, \bar{\Delta}, \varepsilon(, \bar{S}))$, and if the identity $\left(\Psi_{A, A} \circ \bar{\Delta}\right) \cdot \mathcal{R}=\mathcal{R} \cdot \Delta$ holds, where "." is the convolution product and $\bar{A}_{\text {op }}$ is the opposite bialgebra w.r.t. $\bar{A}$ in $(\mathcal{C}, \otimes, \mathbf{1}, \Psi)$. Similar to the case of ordinary quantum groups it is shown in [1] that the antipodes of the quasitriangular Hopf algebra $(A, \bar{A}, \mathcal{R})$ are invertible to each other ${ }^{1}$. In particular $\bar{S}^{-1}=u \cdot S \cdot u^{-1}$, where $u:=m \circ\left(\operatorname{id}_{A} \otimes S\right) \circ \mathcal{R}$.

For a quasitriangular bialgebra $(A, \bar{A}, \mathcal{R})$ we define the category $\mathcal{C}_{\mathcal{O}(A, \bar{A})}$ as the full subcategory of the category of $A$-right modules $\mathcal{C}_{A}$ with objects $\left(X, \mu_{r}\right)$ satisfying the identity

$$
\left(\operatorname{id}_{A} \otimes \mu_{r}\right) \circ\left(\Psi_{X A} \otimes \operatorname{id}_{A}\right) \circ\left(\operatorname{id}_{X} \otimes \Delta\right)=\left(\operatorname{id}_{A} \otimes \mu_{r}\right) \circ\left(\Psi_{X A}^{-1} \otimes \operatorname{id}_{A}\right) \circ\left(\operatorname{id}_{X} \otimes \bar{\Delta}\right) .
$$

This category is identified with a full braided monoidal subcategory of $\mathcal{D} \mathcal{Y}(\mathcal{C})_{A}^{A}[1,12]$; every module $\left(X, \mu_{r}\right)$ in $\mathcal{C}_{\mathcal{O}(A, \bar{A})}$ becomes a crossed module $\left(X, \mu_{r}, \nu_{r}\right)$ in $\mathcal{D} \mathcal{Y}(\mathcal{C})_{A}^{A}$ through the coaction $\nu_{r}:=\left(\mu_{r} \otimes \mathrm{id}_{A}\right) \circ\left(\mathrm{id}_{X} \otimes \mathcal{R}\right)$. This embedding allows us to prove the following generalized bosonization construction [1].

\footnotetext{
${ }^{1}$ This result does not hold in general in [11] since there $\bar{A}_{\mathrm{op}}$ is not necessarily supposed to be a Hopf algebra.
} 
TheOREM 7. Let $\left(A, \bar{A}, \mathcal{R}_{A}\right)$ be a quasitriangular bialgebra in $\mathcal{C}$ and $\left(B, \bar{B}, \mathcal{R}_{B}\right)$ be a quasitriangular bialgebra in $\mathcal{C}_{\mathcal{O}(A, \bar{A})}$ then $\left(A \ltimes B, \bar{A} \ltimes \bar{B}, \mathcal{R}_{A \ltimes B}\right)$ is a quasitriangular bialgebra in $\mathcal{C}$, where $A \ltimes B$ and $\bar{A} \ltimes \bar{B}$ are the bialgebras obtained through the functor $A \ltimes(-)$ and $\bar{A} \ltimes(-)$ in $\mathcal{C}$ and in $\overline{\mathcal{C}}$ respectively. The $R$-matrix of the product is given by

$$
\mathcal{R}_{A \ltimes B}:=\left(m_{A \ltimes B} \otimes m_{A \ltimes B}\right) \circ\left(\iota_{B} \otimes\left(\iota_{A} \otimes \iota_{A}\right) \circ \mathcal{R}_{A} \otimes \iota_{B}\right) \circ \mathcal{R}_{B}
$$

where $\iota_{A}: A \rightarrow A \ltimes B$ and $\iota_{B}: B \rightarrow A \ltimes B$ denote the canonical algebra monomorphisms. Analogous results hold in the Hopf algebra context.

Theorem 7 allows us to specify the braided version of the Radford-Majid theorem to the case where the braided groups are equipped with quasitriangular structures respected by the projections in the following sense.

Definition 5. Let $\left(A, \bar{A}, \mathcal{R}_{A}\right)$ and $\left(H, \bar{H}, \mathcal{R}_{H}\right)$ be braided quantum groups in $\mathcal{C}$. Then the pair $\left(A \underset{p_{A}}{\stackrel{i_{A}}{\longrightarrow}} H, \bar{A} \underset{p_{\bar{A}}}{\stackrel{i_{\bar{A}}}{\longrightarrow}} \bar{H}\right)$ is called a quantum group projection if the following holds.

1. Both $\left(A, H, i_{A}, p_{A}\right)$ and $\left(\bar{A}, \bar{H}, i_{A}, p_{A}\right)$ are bialgebra projections in $\mathcal{C}$ and $\overline{\mathcal{C}}$ respectively.

2. For $\underline{H} \in \mathrm{Ob}\left({ }_{A}^{A} \mathcal{C}_{A}^{A}\right)$ and $\underline{\bar{H}} \in \mathrm{Ob}\left(\frac{\bar{A}}{\bar{A}} \overline{\mathcal{C}} \frac{\bar{A}}{A}\right)$ according to Theorem 5 the idempotents ${ }_{\underline{H}} \Pi$ 3. and $\underline{\underline{H}} \Pi$ defined in Lemma 2 coincide.

$\left(\operatorname{id}_{H} \otimes p_{A}\right) \circ \mathcal{R}_{H}=\left(i_{A} \otimes \operatorname{id}_{A}\right) \circ \mathcal{R}_{A} \quad$ and $\quad\left(p_{A} \otimes \operatorname{id}_{H}\right) \circ \mathcal{R}_{H}=\left(\operatorname{id}_{A} \otimes i_{A}\right) \circ \mathcal{R}_{A}$.

Then we obtain a Radford-Majid criterion for braided quantum groups.

THEOREM 8. Let $\left(A, \bar{A}, \mathcal{R}_{A}\right) \underset{\left(p_{A}, p_{\bar{A}}\right)}{\stackrel{\left(i_{A}, i_{\bar{A}}\right)}{\longrightarrow}}\left(H, \bar{H}, \mathcal{R}_{H}\right)$ be a braided quantum group projection in $\mathcal{C}$. Then there exists a quantum group $\left(B, \bar{B}, \mathcal{R}_{B}\right)$ in the category $\mathcal{C}_{\mathcal{O}(A, \bar{A})}$ such that

$$
\left(H, \bar{H}, \mathcal{R}_{H}\right) \simeq\left(A \ltimes B, \bar{A} \ltimes \bar{B}, \mathcal{R}_{A \ltimes B}\right) .
$$

4. Braided differential calculus. Differential calculi on quantum groups are constructed in [20] where Hopf bimodules or bicovariant bimodules appear as the basic notion in Woronowicz's approach to bicovariant differential calculi on quantum groups. The differential bialgebra structure of the exterior higher order differential calculus, which has been generated by a first order bicovariant differential calculus, was found in [4] after the more general investigation of differential bialgebras and quantum groups had been worked out in [16]. These authors all have in common that they work over the symmetric tensor category of vector spaces over a field $k$. Braided differential calculi have been considered in [13] on the quantum plane - the fundamental (co-)representation of the underlying (co-)quasitriangular quantum group. A successful attempt to construct braided bicovariant differential calculi is described in [8] where braided $G L_{q}(n)$-covariant differential calculi on the braided matrix algebra $B M_{q}(n)$ and on the quantum hyperplanes have been found. 
In this section we outline the definition and the construction of braided bicovariant differential calculi [3]. We show that every braided first order bicovariant differential calculus over a Hopf algebra $H$ in $\mathcal{C}$ induces a braided exterior bicovariant differential calculus over $H$. The universality of this construction is discussed. For the derivation of these facts we make use of the results of the previous sections. In what follows we focus our consideration to abelian, braided monoidal categories with a bilinear tensor product, such that $X$ is flat for every object $X$ in the category. We will call henceforth categories with these properties $* *$-abelian categories. All the results in the sequel can be derived under weaker conditions. The more general case is studied in [3].

Remark 1 . If the category $\mathcal{C}$ of the previous sections is $* *$-abelian then it is a nontrivial but straightforward computation to verify that in particular the category ${ }_{H}^{H} \mathcal{C}_{H}^{H}$ is also $* *$-abelian.

Let us consider any $* *$-abelian category $\mathcal{D}$. Let in the following $I$ be either the set $\{0,1\}$ or $\mathbb{I} N_{0}$. Then the $I$-graded category $\mathcal{D}^{I}$ is the functor category where $I$ is considered to be discrete, i.e. the objects in $\mathcal{D}^{I}$ are of the form $\hat{X}=\left(X_{0}, X_{1}, \ldots\right)$ where $X_{j} \in \mathrm{Ob}(\mathcal{D})$ and the morphisms $\hat{f}: \hat{X} \rightarrow \hat{Y}$ are given by $\hat{f}=\left(f_{0}, f_{1}, \ldots\right)$ where $f_{j}: X_{j} \rightarrow Y_{j}$ is a morphism in $\mathcal{D}$ (see e.g. [14]). It is not difficult to verify

Proposition 9. $\mathcal{D}^{I}$ is a**-abelian category. In particular the tensor product and the braiding are given respectively by $(\hat{X} \otimes \hat{Y})_{j}=\bigoplus_{k+l=j} X_{k} \otimes Y_{l}$ and $\left(\hat{\Psi}_{\hat{X} \hat{Y}}\right)_{j}=$ $\bigoplus_{k+l=j}(-1)^{k l} \Psi_{X_{k} Y_{l}}$.

The category of complexes over $\mathcal{D}$ will be defined in the usual manner - see e.g. [7].

Definition 6. $(\hat{X}, \hat{\mathrm{d}})$ is called a complex in $\mathcal{D}^{I}$ if $\hat{X}$ is an object in $\mathcal{D}^{I}$ and the differential $\hat{\mathrm{d}}=\left(\mathrm{d}_{0}, \mathrm{~d}_{1}, \ldots\right)$ is given as a sequence of morphisms $\mathrm{d}_{j}: X_{j} \rightarrow X_{j+1}$ in $\mathcal{D}$ such that $\mathrm{d}_{j+1} \circ \mathrm{d}_{j}=0$. Morphisms of complexes $\hat{f}:\left(\hat{X}, \hat{\mathrm{d}}_{X}\right) \rightarrow\left(\hat{Y}, \hat{\mathrm{d}}_{Y}\right)$ are morphisms in $\mathcal{D}^{I}$ such that $f_{j+1} \circ \mathrm{d}_{X j}=\mathrm{d}_{Y j} \circ f_{j}$. The category of complexes is denoted by ${ }^{c} \mathcal{D}^{I}$.

Proposition 10. ${ }^{c} \mathcal{D}^{I}$ is a**-abelian category. The tensor product and the braiding are given as in Proposition 9. The differential of the tensor product of two complexes is given through

$$
\left(\mathrm{d}_{\hat{X} \otimes \hat{Y}}\right)_{j}=\sum_{k+l=j}\left(\mathrm{~d}_{X k} \otimes \mathrm{id}_{Y_{l}}+(-1)^{k} \mathrm{id}_{X_{k}} \otimes \mathrm{d}_{Y_{l}}\right)
$$

Through the canonical mappings $X \mapsto \hat{X}:=(X, 0,0, \ldots) \mapsto(\hat{X}, \hat{\mathrm{d}}:=0)$ one obtains the following braided monoidal inclusions.

LEMMA 11.

$$
\mathcal{D} \hookrightarrow \mathcal{D}^{I} \hookrightarrow{ }^{c} \mathcal{D}^{I}
$$

Let $H$ be a Hopf algebra in $\mathcal{D}$ (with invertible antipode). Then by use of Remark 1 and Lemma 11 we obtain

Proposition 12. There are braided monoidal isomorphisms

$$
{ }_{H}^{H}\left(\mathcal{D}^{I}\right)_{H}^{H} \cong\left({ }_{H}^{H} \mathcal{D}_{H}^{H}\right)^{I} \quad \text { and } \quad{ }_{H}^{H}\left({ }^{c} \mathcal{D}^{I}\right)_{H}^{H} \cong{ }^{c}\left({ }_{H}^{H} \mathcal{D}_{H}^{H}\right)^{I} .
$$


For any object $X$ in $\mathcal{D}$ we consider its tensor algebra $T_{\mathcal{D}}(X)$ in $\mathcal{D}^{I N_{0}}$ with $\left(T_{\mathcal{D}}(X)\right)_{0}=$ $\mathbf{1}_{\mathcal{D}}$ and $\left(T_{\mathcal{D}}(X)\right)_{j}=X^{\otimes j}$ the $j$-fold tensor product of $X$. Since $\mathcal{D}$ is braided it is possible for any fixed $j \in I$ to define a section $S_{j} \ni \sigma \mapsto \sigma_{\mathcal{D}}(X) \in \operatorname{Aut}\left(X^{\otimes j}\right)$ of the symmetric group $S_{j}$ to the representation of the braid group $B_{j}$ on $X^{\otimes j}$ generated by the braiding $\Psi_{X X}[20]$. We denote by $S_{(k, l)}$ the shuffle permutations in $S_{k+l}$ which shuffle $k$ numbers in $\{1,2, \ldots, k+l\}$ to the first $k$ places without changing their order and the remaining $l$ numbers to the places $k+1, \ldots, k+l$ without changing their orders. Then we define $A_{j}(X)=\sum_{\sigma \in S_{j}}(-1)^{l(\sigma)} \sigma_{\mathcal{D}}(X)$ and $A_{(k, l)}(X)=\sum_{\sigma \in S_{(k, l)}}(-1)^{l(\sigma)} \sigma_{\mathcal{D}}(X)$ where $l(\sigma)$ is the length of the permutation $\sigma$. Similarly we define

$$
A^{(k, l)}(X)=\sum_{\sigma \in S^{(k, l)}}(-1)^{l(\sigma)} \sigma_{\mathcal{D}}(X)
$$

where $S^{(k, l)}$ are the shuffle permutations inverse to $S_{(k, l)}$. Using braid algebra one can prove the following proposition.

Proposition 13. On $T_{\mathcal{D}}(X)$ a Hopf algebra structure is given by

$$
\begin{aligned}
\mathrm{m}_{(n, m)} & \cong \operatorname{id}_{X \otimes n+m}: X^{\otimes n} \otimes X^{\otimes m} \rightarrow X^{\otimes n+m}, \\
\eta_{0} & =\operatorname{id}_{k}, \eta_{j}=0 \text { for } j \neq 0, \\
\Delta_{(n, m)} & \cong A_{(n, m)}(X): X^{\otimes n+m} \rightarrow X^{\otimes n} \otimes X^{\otimes m}, \\
\varepsilon_{0} & =\operatorname{id}_{k}, \varepsilon_{j}=0 \text { for } j \neq 0, \\
S_{j} & =(-1)^{j}\left(\sigma_{j}^{0}\right)_{\mathcal{D}}(X)
\end{aligned}
$$

where $\sigma_{j}^{0}=\left(\begin{array}{lll}1 & \ldots & j \\ j & \ldots & 1\end{array}\right)$. Because of duality a Hopf structure dual to (18) can be established on $T_{\mathcal{D}}(X)$. Explicitely it is given by

$$
\begin{aligned}
\stackrel{\circ}{\mathrm{m}}_{(n, m)} & \cong A^{(n, m)}(X): X^{\otimes n} \otimes X^{\otimes m} \rightarrow X^{\otimes n+m}, \\
\stackrel{\circ}{\eta}_{0} & =\mathrm{id}_{k}, \stackrel{\circ}{\eta}_{j}=0 \text { for } j \neq 0, \\
\stackrel{\circ}{\Delta}_{(n, m)} & \cong \mathrm{id}_{X} \otimes n+m \\
\stackrel{\circ}{\varepsilon}_{0} & =\mathrm{id}_{k}, \stackrel{\circ}{\varepsilon}_{j}=0 \text { for } j \neq 0, \\
\stackrel{\circ}{S}_{j} & =(-1)^{j}\left(\sigma_{j}^{0}\right)_{\mathcal{D}}(X) .
\end{aligned}
$$

The dual Hopf algebra according to (19) will be denoted by $\stackrel{\circ}{T}_{\mathcal{D}}(X)$.

The two Hopf algebras are linked in a nice way via the graded morphism $(\hat{A}(X))_{j}:=$ $A_{j}(X): X^{\otimes j} \rightarrow X^{\otimes j}$. This yields the following proposition [3].

Proposition 14. $\hat{A}(X): T_{\mathcal{D}}(X) \rightarrow \stackrel{\circ}{T}_{\mathcal{D}}(X)$ with $(\hat{A}(X))_{j}:=A_{j}(X)$ is a bialgebra morphism in $\mathcal{D}^{I}$. Hence it follows that $\hat{A}(X)$ induces a Hopf algebra structure in $\mathcal{D}^{I}$ on the object

$$
T_{\mathcal{D}}^{\wedge}(X):=\operatorname{coim}(\hat{A}(X)) .
$$

Remark 2. If $\mathcal{D}$ is a category of vector spaces then $T_{\mathcal{D}}^{\wedge}(X)$ is a braided version of the exterior tensor algebra, $T_{\mathcal{D}}^{\wedge}(X)=T_{\mathcal{D}} /(\operatorname{ker} \hat{A}(X))$. 
From now on we suppose that the category $\mathcal{C}$ of the previous sections is $* *$-abelian. The definition of a differential calculus in $\mathcal{C}$ is then given by

Definition 7. A complex $(\hat{Y}, \hat{\mathrm{d}})$ in ${ }^{c} \mathcal{C}^{I}$ is called a differential calculus if $(\hat{Y}, \hat{\mathrm{d}})$ is an algebra in ${ }^{c} \mathcal{C}^{I}$ and the image of the morphism $\mathrm{m}_{0, j+1} \circ\left(\mathrm{id}_{Y_{0}} \otimes \mathrm{d}_{j}\right)$ is $Y_{j+1}$ for all $j \in I$. Let $\hat{\mathcal{B}}$ be a bialgebra in $\mathcal{C}^{I}$. Then $(\hat{Y}, \hat{\mathrm{d}})$ is called $\hat{\mathcal{B}}$-left covariant, $\hat{\mathcal{B}}$-right covariant or $\hat{\mathcal{B}}$-bicovariant differential calculus if it is a differential calculus in the category ${ }^{\hat{\mathcal{B}}}\left({ }^{c} \mathcal{C}^{I}\right)$, $\left({ }^{c} \mathcal{C}^{I}\right)^{\hat{\mathcal{B}}}$ or ${ }^{\hat{\mathcal{B}}}\left({ }^{c} \mathcal{C}^{I}\right)^{\hat{\mathcal{B}}}$ respectively. ${ }^{2}$

If $I=\{0,1\}$ we sometimes use the name first order differential calculus. If $I=\mathbb{N} N_{0}$ we speak of higher order differential calculus [20]. These definitions generalize the notations of $[20]$ in the following sense. If $(\hat{Y}, \hat{\mathrm{d}})$ is a differential calculus and a bialgebra in ${ }^{c} \mathcal{C}^{I}$ then it is straightforward to show that $(\hat{Y}, \hat{\mathrm{d}})$ is a braided $Y_{0}$-bicovariant differential calculus or a braided bicovariant differential calculus over $Y_{0}$. For $I=\{0,1\}$ this is just a braided version of the definition of bicovariant differential calculi given in [20].

Now let $\mathcal{D}={ }_{H}^{H} \mathcal{C}_{H}^{H}$ be the category of $H$-Hopf bimodules and $((H, X), d)$ be a braided bicovariant first order differential calculus over the Hopf algebra $H$. Then it follows rather immediately that $X$ is in particular an $H$-Hopf bimodule. Hence we can apply the results of Proposition 13 and 14 to derive the Hopf algebra $T_{H}^{\wedge} \mathcal{C}_{H}^{H}(X)$ in $\left({ }_{H}^{H} \mathcal{C}_{H}^{H}\right)^{\mathbb{N}_{0}}$. To obtain from $T_{H}^{\wedge} \mathcal{C}_{H}^{H}(X)$ a Hopf algebra (projection) in $\mathcal{C}^{\mathbb{N}_{0}}$ we apply the corresponding functor of Theorem 5 and call the resulting object $X^{\wedge}$. This object $X^{\wedge}$ turns out to be the braided generalization of an algebra of exterior forms over the group as explained in [20] for the case of the symmetric category of vector spaces. To find the differential structure on $X^{\wedge}$ is a nontrivial matter [3]. We use ideas of [20] and exploit strongly the results and techniques of [2] which have been outlined in the previous sections. We state the result in the following theorem.

THEOREM 15. On $X^{\wedge}$ there exists a unique differential $\hat{\mathrm{d}}$ such that

1. The 0.th component of $\hat{\mathrm{d}}$ is the differential $\mathrm{d}$ of the bicovariant first order differential calculus $(H, X, \mathrm{~d})$, i.e. $\mathrm{d}_{0}=\mathrm{d}$.

2. $\left(X^{\wedge}, \hat{\mathrm{d}}\right)$ is an $\mathbb{N}_{0}$-graded Hopf algebra differential calculus over $H$ and hence in particular a bicovariant differential calculus over $H$.

The construction of $X^{\wedge}$ is universal in the following sense. Consider bialgebras $\hat{Y}$ in $\mathcal{C}^{\mathbb{N} N_{0}}$ such that $Y_{0}$ is a Hopf algebra with invertible antipode and the components $Y_{n+1}$ are generated through the multiplication $\mathrm{m}^{Y}$ by $Y_{0}$ and $Y_{1}$, i.e. $\operatorname{im}\left(m_{1, n}^{Y}\right)=Y_{n+1} \forall n>1$. Then we obtain the next proposition.

Proposition 16. Let $\hat{Y}$ be a bialgebra of the above mentioned form, and let $\left(f_{0}, f_{1}\right)$ : $\left(Y_{0}, Y_{1}\right) \rightarrow\left(H, X_{1}\right)$ be a bialgebra morphism in $\mathcal{C}^{(0,1)}$. Then there exists a unique bialgebra morphism $\hat{f}: \hat{Y} \rightarrow X^{\wedge}$ such that $\hat{f}_{0}=f_{0}$ and $\hat{f}_{1}=f_{1}$.

\footnotetext{
${ }^{2}$ According to the general notion these are the corresponding $\hat{\mathcal{B}}$-comodule categories.
} 


\section{References}

[1] Yu. Bespalov, Crossed Modules and Quantum Groups in Braided Categories, to appear in Appl. Categorical Structures.

[2] Yu. Bespalov and B. Drabant, Hopf (Bi-)Modules and Crossed Modules in Braided Monoidal Categories, preprint UVA-FWI 95-18 (1995).

[3] Yu. Bespalov and B. Drabant, Differential Calculus in Braided Abelian Categories, in preparation.

[4] T. Brzezinski, Remarks on Bicovariant Differential Calculi and Exterior Hopf Algebras, Lett. Math. Phys. 27, 287 (1993).

[5] B. Drabant, Braided Bosonization and Inhomogeneous Quantum Groups, Preprint UVAFWI 94-28 (1994), to appear in Acta Math. Appl.

[6] P. Freyd and D. Yetter, Braided compact closed categories with applications to low dimensional topology, Adv. Math. 77, 156 (1989).

[7] D. Husemoller, Cyclic Homology, Tata Institute Lecture Notes on Mathematics 83, Springer (1991).

[8] A.P. Isaev and A.A. Vladimirov, $G L_{q}(n)$-Covariant Braided Differential Bialgebras, Lett. Math. Phys. 33, 297 (1995).

[9] A. Joyal and R. Street, Braided monoidal categories, Mathematics Reports 86008, Macquarie University (1986).

[10] S. Majid, Algebras and Hopf algebras in braided categories, Advances in Hopf Algebras, Lecture Notes in Pure and Appl. Math. 158, 55, Dekker (1994).

[11] S. Majid, Cross Products by Braided Groups and Bosonization, J. Algebra 163, 165 (1994).

[12] S. Majid, Transmutation theory and rank for quantum braided groups, Math. Proc. Camb. Phil. Soc. 113, 45 (1993).

[13] S. Majid, Free Braided Differential Operators, Braided Binomial Theorem, and the Braided Exponential Map, J. Math. Phys. 34, 4843 (1993).

[14] S. MacLane, Categories. For the Working Mathematician, GTM 5, Springer (1972).

[15] S. MacLane, Natural associativity and commutativity, Rice University Studies 49, 28 (1963).

[16] Yu. I. Manin, Notes on Quantum Groups and Quantum de Rham Complexes, J. Theor. and Math. Phys. 92, 425 (1992).

G. Maltsiniotis, Le langage des espaces et des groupes quantiques, Commun. Math. Phys. 151, 275 (1993).

[17] D.E. Radford, The Structure of Hopf Algebras with a Projection, J. Algebra 92, 322 (1985).

[18] D.E. Radford and J. Towber, Yetter-Drinfel'd categories associated to an arbitrary bialgebra, J. Pure Appl. Algebra 87, 259 (1993).

[19] M.E. Sweedler, Hopf algebras, W.A. Benjamin, New York (1969).

[20] S.L. Woronowicz, Differential Calculus on Compact Matrix Pseudogroups (Quantum Groups), Commun. Math. Phys. 122, 125 (1989).

[21] D. Yetter, Quantum groups and representations of monoidal categories, Math. Proc. Camb. Phil. Soc. 108, 261 (1990). 